\title{
Wavelength-tunable mode-locked fiber laser with birefringence-enhanced cavity
}

\author{
Chuanhang Zou ${ }^{1}$, Tianxing Wang ${ }^{1}$, Zhijun Yan², Qianqian Huang ${ }^{1}$, Mohammed AlAraimi ${ }^{3,4,5}$, \\ Aleksey Rozhin ${ }^{3,4}$, Chengbo Mou, ${ }^{1, *}$ \\ ${ }^{1}$ Key Laboratory of Specialty Fiber Optics and Optical Access Networks, Shanghai University, Shanghai \\ 200072, P. R. China \\ ${ }^{2}$ School of Optical and Electronic Information, National Engineering Laboratory for Next Generation Internet \\ Access System, Huazhong University of Science and Technologies, Wuhan 430074, P.R.China \\ ${ }^{3}$ Aston Institute of Photonic Technologies (AIPT), Aston University, Birmingham, B4 7ET, United Kingdom \\ ${ }^{4}$ Nanoscience Research Group, Aston University, Birmingham, B4 7ET, United Kingdom \\ ${ }^{5}$ Al Musanna College of Technology, Muladdah, Al Musanna, P.O.Box 191, P.C.314, Sultanate of Oman \\ *mouc1@shu.edu.cn
}

\begin{abstract}
A wavelength-tunable Erbium-doped fiber laser with birefringence-enhanced cavity is proposed. A $45^{\circ}$ tiled fiber grating $\left(45^{\circ} \mathrm{TFG}\right)$ and a section of polarization maintaining (PM) fiber are placed between two two polarization controllers (PCS). Two PCs and PM fiber are employed to enhance the birefringence effect. The $45^{\circ}$ TFG plays the role of an in-fiber polarizer. The fiber laser is mode-locked by single walled carbon nanotube polyvinyl alcohol (SWCNTs-PVA) composite film. Central wavelength of the fiber laser can be tuned from $1552.52 \mathrm{~nm}$ to $1575.28 \mathrm{~nm}$ with a tuning range of $22.76 \mathrm{~nm}$. The demonstrated laser provided the widest tuning range in a high repetition rate all-fiber Erbium doped laser based on SWCNT.
\end{abstract}

Keywords: mode locked fiber laser, tilted fiber grating, wavelength tuning, carbon nanotube

\section{INTRODUCTION}

During the past decade, wavelength-tunable mode-locked fiber laser have attract a great deal of attention owing to their application in many fields such as spectroscopy, fiber-optic sensors and optical telecommunications, optical instrumentation [1-4]. Up to now, many methods have been proposed to realize wavelength tunable mode-locked laser. The central wavelength can be tuned by adding a MachZehnder interferometer or acousto-optic tunable filter in the laser [5,6]. Fiber gratings such as chirped fiber Bragg grating [7], W-shape long period grating (LPG) [8] are also employed to tune the wavelength of the fiber laser. Commercial mechanically tunable bandpass filter are also shown to be a good device for achieving wavelength tuning of the laser $[9,10]$.Another method is to use the intrinsic birefringence effect of the laser as a birefringent filter to tune the wavelength [11].In order to enhance the birefringence effect of laser, the PM fiber with appropriate length can be incorporated into the laser cavity [12].
In this report, we proposed a wavelength-tunable carbon nanotube mode-locked fiber laser with birefringence-enhanced cavity. Wavelength can be tuned by a birefringent filter formed by two PCs, $45^{\circ} \mathrm{TFG}, \mathrm{PM}$ fiber. The $45^{\circ} \mathrm{TFG}$ enables the $p$-light to propagate along the fiber core with a small loss, while $s$-light is coupled into the cladding along the direction perpendicular to the fiber core. So $45^{\circ} \mathrm{TFG}$ plays the role of an in-fiber polarizer [13]. Two PCs are used to optimize the polarization state and adjust the birefringence. PM fiber is used to enhance birefringence.

\section{EXPERIMENTAL SETUP}

The fiber laser is mode-locked by SWCNTs-PVA composite film with $6.2 \%$ modulation depth. Detailed fabrication of SWCNTs-PVA composite film can be found in [14]. $45^{\circ} \mathrm{TFG}$ used in fiber laser has $-7.23 \mathrm{~dB}$ insertion loss and $19 \mathrm{~dB}$ polarization dependent loss (PDL) at $1550 \mathrm{~nm}$. Fig.1.(a)\&(b) shows the measured insertion loss and PDL of the $45^{\circ} \mathrm{TFG}$, respectively. The fabrication of $45^{\circ} \mathrm{TFG}$ is described in detail in ref[15].

Schematic of the wavelength-tunable fiber laser is shown in Fig.2. The fiber laser is pumped by a $980 \mathrm{~nm}$ benchtop laser (OV LINK) with maximum pump power of $611 \mathrm{~mW}$ via a WDM with pigtail of $2.47 \mathrm{~m}$ OFS980 which has normal dispersion $\beta 2=+4.5 \mathrm{ps} 2 / \mathrm{km}$. A section of Erbium-doped fiber (EDF Er80-8/125 from Liekki) with length of $1.1 \mathrm{~m}$ and group velocity dispersion (GVD) of $-20 \mathrm{ps}^{2} / \mathrm{km}$ is used as active fiber of the fiber laser. Two PCs are used to adjust the polarization. A $45^{\circ} \mathrm{TFG}$ and a section of PM fiber with length of $16 \mathrm{~cm}$ are placed between the two PCs. SWCNTs-PVA composite film is sandwiched between two standard fiber connector ferrules. An optical isolator (OSI) is employed to prevent the reverse light and ensure unidirectional operation of the fiber laser. $40 \%$ of the light is coupled out by a $60: 40$ output coupler. The laser also includes $7.1 \mathrm{~m}$ single mode fiber (SMF) with anomalous dispersion $\beta_{2}=-22.8 \mathrm{ps} 2 / \mathrm{km}$. Total cavity length is $10.7 \mathrm{~m}$ and net dispersion is $-0.376 \mathrm{ps}^{2}$, so it is shown that the laser operates in the soliton region. $40 \%$ output pulses of the fiber is divided into two parts by a coupler, then an optical spectrum 
analyzer (OSA, Yokogawa AQ6370C) is employed to analyze the output spectrum and a $1 \mathrm{GHz}$ mixed signal oscilloscope (OSC, Tektronix MSO4104) and a spectrum analyzer (SIGLENT, SSA 3032X) with a $12.5 \mathrm{GHz}$ high speed biased photodetector (Newport, $818-\mathrm{BB}-51 \mathrm{~F})$ are used to record the pulse trains and radio frequency $(\mathrm{RF})$ spectrum of pulses.
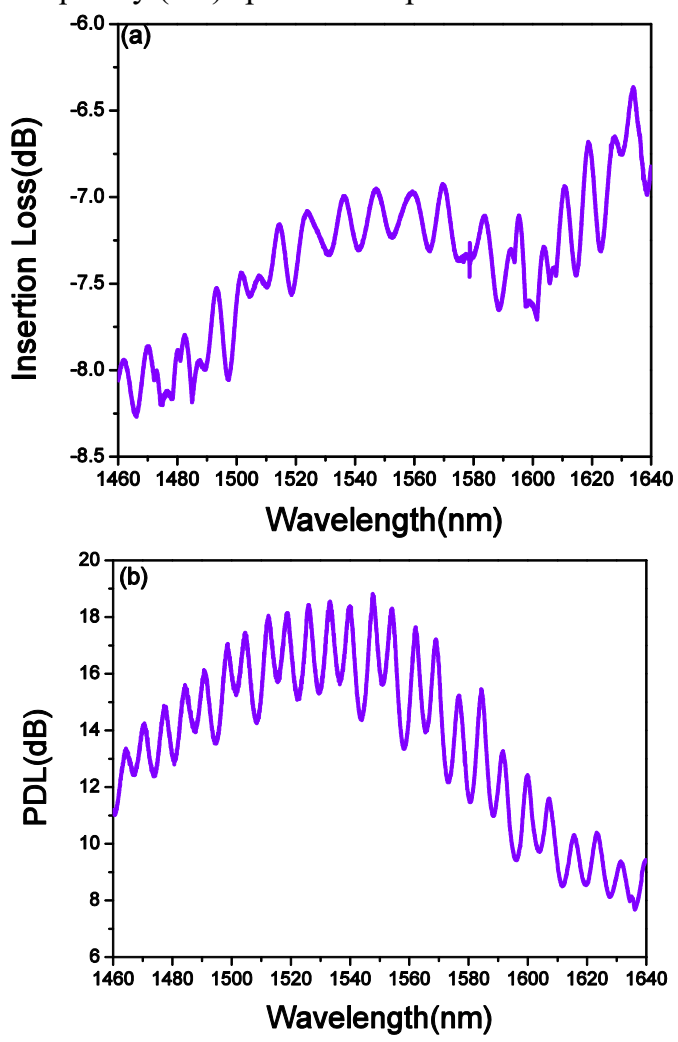

Fig.1. Measured (a) insertion loss, (b) PDL of $45^{\circ} \mathrm{TFG}$

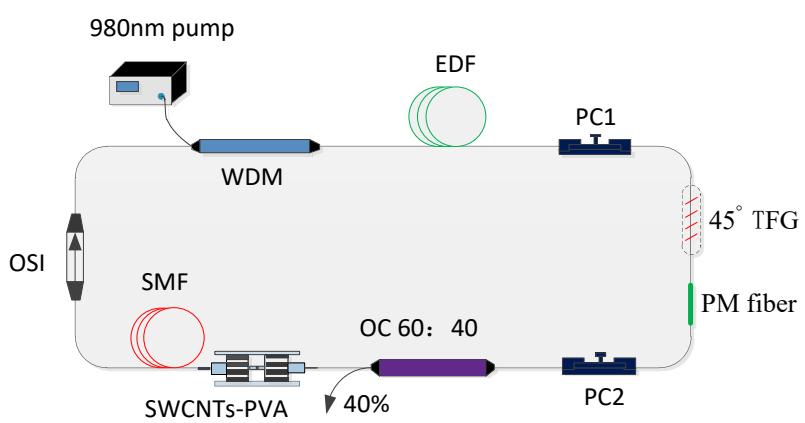

Fig.2. Schematic of the wavelength-tunable fiber laser

\section{EXPERIMENTAL RESULTS}

The mode-locked threshold of the laser is $220 \mathrm{~mA}$. The laser is mode-locked by SWCNTs-PVA composite film without nonlinear polarization evolution (NPE), because only when the pump current is greater than $500 \mathrm{~mA}$, NPE will work. When the pump current is increased to $260 \mathrm{~mA}$, adjusting the polarization controller slightly, the laser will operate in a very stable mode locking state. Fig.3. (a) shows the pulse trains of the fiber laser with pulse interval of $51.5 \mathrm{~ns}$ under pump current of $260 \mathrm{~mA}$.
RF spectrum of pulses is depicted in Fig.3.(b). We can see that the signal to noise ratio (SNR) at $19.4 \mathrm{MHz}$ is $50.8 \mathrm{~dB}$. Both the pulse trains and RF spectrum indicate that the fiber operates in single pulse state and the pulses is relatively stable. At this point, the central wavelength of the fiber laser can be tuned continuously by adjusting the PCs. From Fig.4.(a), we can see that the central wavelength can be tuned from $1552.52 \mathrm{~nm}$ to $1575.28 \mathrm{~nm}$ with a tuning range of $22.76 \mathrm{~nm}$. In the wavelength tuning process, the laser has always been working in single pulse state. Pulse duration at different central wavelength are also measured. Fig.4.(b) shows autocorrelation traces of laser output at different central wavelengths. The minimum pulse width is $1.23 \mathrm{ps}$ corresponding to central wavelength of $1570.01 \mathrm{~nm}$ and the maximum pulse width is about $3.48 \mathrm{ps}$ corresponding to the central wavelength of $1567.67 \mathrm{~nm}$. The minimum spectral width and output power are $1.28 \mathrm{~nm}, 0.22 \mathrm{~mW}$ respectively. The maximum spectral width and output power are $2.88 \mathrm{~nm}, 0.76 \mathrm{~mW}$ respectively. Further increasing the pump current, the central wavelength of the laser also can be tuned, but the range is small and laser will become unstable.
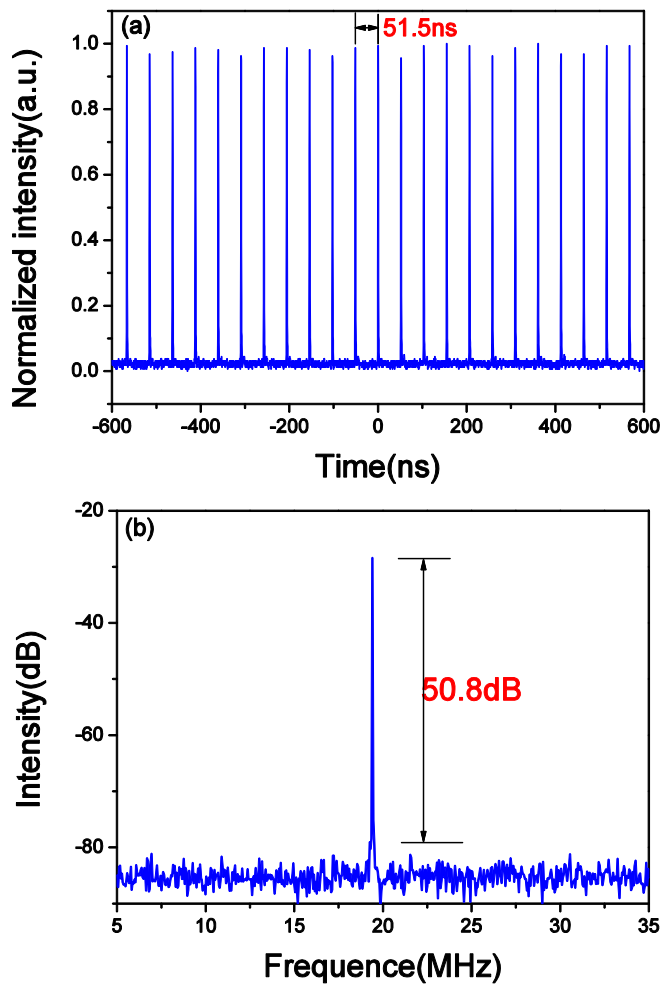

Fig.3. Measured (a) pulse trains, (b) RF spectrum of pulses. 

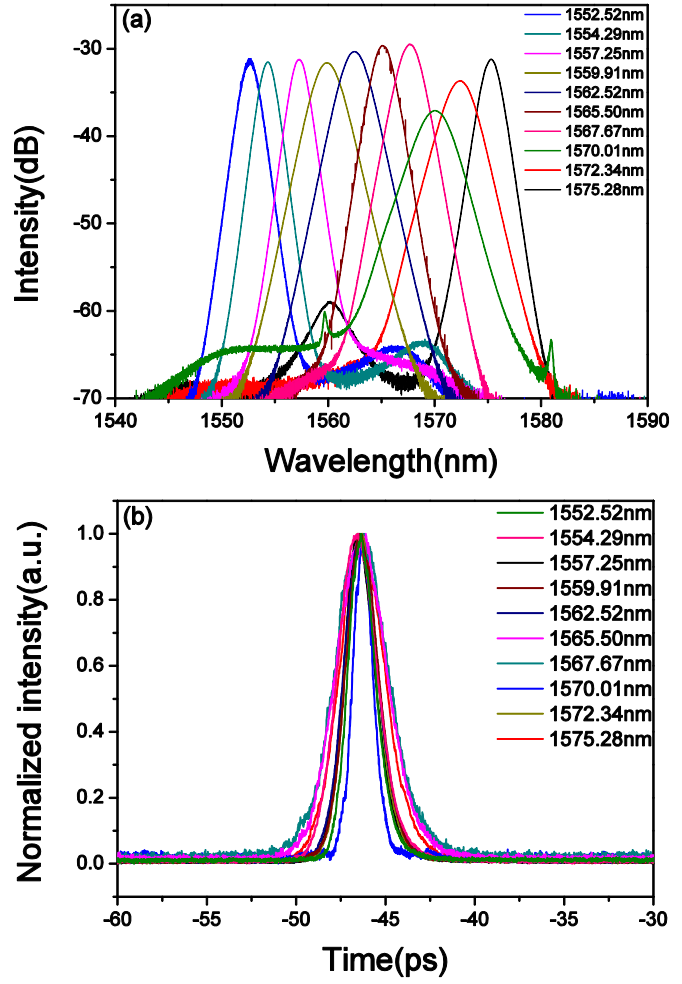

Fig.4. Measured characteristics of tunable mode-locked laser (a) Spectral evolution, (b) Autocorrelation traces of laser output at different central wavelengths.

\section{CONCLUSIONS}

We propose and demonstrate a wavelength-tunable erbium-doped fiber laser with birefringence-enhanced cavity. The fiber laser is mode-locked by SWCNTs-PVA composite film. Central wavelength of the fiber laser can be tuned from $1552.52 \mathrm{~nm}$ to $1575.28 \mathrm{~nm}$ with tunable range of $22.76 \mathrm{~nm}$. The minimum pulse width is $1.23 \mathrm{ps}$ corresponding central wavelength $1570.01 \mathrm{~nm}$. The maximum spectral width and output power are $2.88 \mathrm{~nm}$, $0.76 \mathrm{~mW}$ corresponding central wavelength $1572.34 \mathrm{~nm}$, $1567.67 \mathrm{~nm}$ respectively. The demonstrated laser provided the widest tuning range in a high repetition rate all-fiber Erbium doped laser based on SWCNT.

\section{ACKNOWLEDGMENTS}

This work was supported by the National Natural Science Foundation of China (NSFC) (61605107, 61505244) and the Young Eastern Scholar program (QD2015027) at Shanghai Institutions of Higher Learning and the "Young 1000 Talent Plan" program of China.

\section{REFERENCES}

[1] Ailing Zhang, Heliang Liu , M.S. Demokan, H.Y. Tam . Stable and broad bandwidth multiwavelength fiber ring laser incorporating a highly nonlinear photonic crystal fiber. IEEE photonics technology letters, vol.17, Issue 12, pp.2535-2537, November 2005.
[2] Rosa Ana Perez-Herreraa, Montserrat Fernandez-Vallejoa, Silvia Diaza, M. Angeles Quintelab, Manuel Lopez-Amoa, José Miguel López-Higuera. Stability comparison of two quadruple-wavelength switchable erbium-doped fiber lasers. vol.16, Issue 4, pp.205-211, August 2010.

[3] Oleg Okhotniko, Anatoly Grudinin, Markus Pessa. Ultra-fast fibre laser systems based on SESAM technology: new horizons and applications. New journal of physics, vol.6, Issue 1, pp.177, November 2004.

[4] O. G. Okhotnikov, L. Gomes, N. Xiang, T. Jouhti, A. B. Mode-locked ytterbium fiber laser tunable in the 980 1070-nm spectral range. Optics letters, vol.28, Issue 17, pp.1522-1524, 2003.

[5] Guolu Yin, Xiaozhen Wang, and Xiaoyi Bao. Effect of beam waists on performance of the tunable fiber laser based on in-line two-taper Mach-Zehnder interferometer filter. Applied optics, vol.50, Issue 29, pp.5714-5720, 2011.

[6] Min-Yong Jeon, Hak Kyu Lee , Kyong Hon Kim , El-Hang Lee, Seok Hyun Yun, Byoung Yoon Kim, Yeon Wan Koh. An electronically wavelength-tunable mode-locked fiber laser using an all-fiber acoustooptic tunable filter. IEEE Photonics Technology Letters, vol.8, Issue 12, pp.1618-1620, 1996.

[7] Xiaoying He, Zhi-bo Liu, and D. N. Wang, passively mode-locked fiber laser based on graphene and chirped fiber Bragg grating. Optics letters, vol.37, Issue 12, pp.2394-2396, 2012.

[8] Jie Wang, A. Ping Zhang, Yong Hang Shen, Hwa-yaw Tam, and P. K. A. Wai. Widely tunable mode-locked fiber laser using carbon nanotube and LPG W-shaped filter[J]. Optics letters, vol.40, Issue 18, pp.4329-4332, 2015.

[9] Zhipei Sun, Daniel Popa, Tawfique Hasan, Felice Torrisi, Fengqiu Wang, Edmund J. R. Kelleher, John C. Travers, Valeria Nicolosi, and Andrea C. Ferrari. A stable, wideband tunable, near transform-limited, graphene-mode-locked, ultrafast laser. Nano Research, vol.3, Issue 9, pp.653-660, 2010.

[10] F. Wang, A. G. Rozhin, V. Scardaci, Z. Sun, F. Hennrich, I. H. White, W. I. Milne and A. C. Ferrari. Wideband-tunable, nanotube mode-locked, fibre laser. Nature nanotechnology, vol.3, Issue 12, pp.738-742, 2008.

[11] Han Zhang, Dingyuan Tang, R. J. Knize, Luming Zhao, Qiaoliang Bao, and Kian Ping Loh. Graphene mode locked, wavelength-tunable, dissipative soliton fiber laser. Applied Physics Letters, vol.96, Issue 11, pp. 111112, 2010.

[12] Z. X. Zhang, Z. W. Xu, and L. Zhang. Tunable and switchable dual-wavelength dissipative soliton generation in an all-normal-dispersion Yb-doped fiber laser with birefringence fiber filter. Optics express, vol.20, Issue 24, pp.26736-26742, 2012.

[13] Chengbo Mou, Kaiming Zhou, Lin Zhang, and Ian Bennion. Characterization of $45^{\circ}$-tilted fiber grating and its polarization function in fiber ring laser. JOSA B, vol.26, Issue 10, pp. 1905-1911, 2009.

[14] C. Mou, S. Sergeyev, A. Rozhin, and S. Turistyn. All-fiber polarization locked vector soliton laser using carbon nanotubes. Optics letters, Vol.36, Issue 19, pp. 3831-3833, 2011.

[15] Kaiming Zhou, George Simpson, Xianfeng Chen, Lin Zhang, and Ian Bennion. High extinction ratio in-fiber polarizers based on 45 tilted fiber Bragg gratings. Vol.30, Issue 11, pp.1285-1287, 2005. 